\title{
e-VLBI Technology in VLBI Network "Quasar”
}

\section{Andrey Finkelstein}

Institute of Applied Astronomy Russian Academy of Sciences

Kutuzova 10, 191187, Saint-Petersburg, Russia

E-mail: amfeipa.nw.ru

\section{Alexander Ipatov}

Institute of Applied Astronomy Russian Academy of Sciences Kutuzova 10, 191187, Saint-Petersburg, Russia

E-mail: avi@ipa.nw.ru

\section{Michael Kaidanovsky}

Institute of Applied Astronomy Russian Academy of Sciences Kutuzova 10, 191187, Saint-Petersburg, Russia

E-mail:kmneipa.nw.ru

\section{Ilia Bezrukov}

Institute of Applied Astronomy Russian Academy of Sciences Kutuzova 10, 191187, Saint-Petersburg, Russia

E-mail:bia@ipa.nw.ru

\section{Andrey Mikhailov}

Institute of Applied Astronomy Russian Academy of Sciences Kutuzova 10, 191187, Saint-Petersburg, Russia

E-mail:agmeipa.nw.ru

\section{Alexander Salnikov ${ }^{1}$}

Institute of Applied Astronomy Russian Academy of Sciences Kutuzova 10, 191187, Saint-Petersburg, Russia

E-mail:ais@ipa.nw.ru

\section{Igor Surkis}

Institute of Applied Astronomy Russian Academy of Sciences Kutuzova 10, 191187, Saint-Petersburg, Russia

E-mail:surkis @ipa.nw.ru

\section{Elena Skurikhina}

Institute of Applied Astronomy Russian Academy of Sciences Kutuzova 10, 191187, Saint-Petersburg, Russia E-mail:seaeipa.nw.ru

\section{A. Salnikov}


The development of technical facilities in Russia makes it possible to apply the e-VLBI technology to the VLBI Network "Quasar". Some experiments were made for the transmission of Intensive VLBI-sessions data from the "Badary" and "Zelenchukskaya" observatories to the Control and Processing Center in the Institute of Applied Astronomy. An Intensive session of $\sim$ 40GB was transmitted over shared networks using Tsunami-UDP protocol.

Science and Technology of Long Baseline Real-Time Interferometry:

The 8th International e-VLBI Workshop - EXPReS09

Madrid, Spain

June 22-26, 2009 


\section{Introduction}

Institute of Applied Astronomy of the Russian Academy of Sciences carries out regular observations on the VLBI Network "Quasar" (Fig. 1).

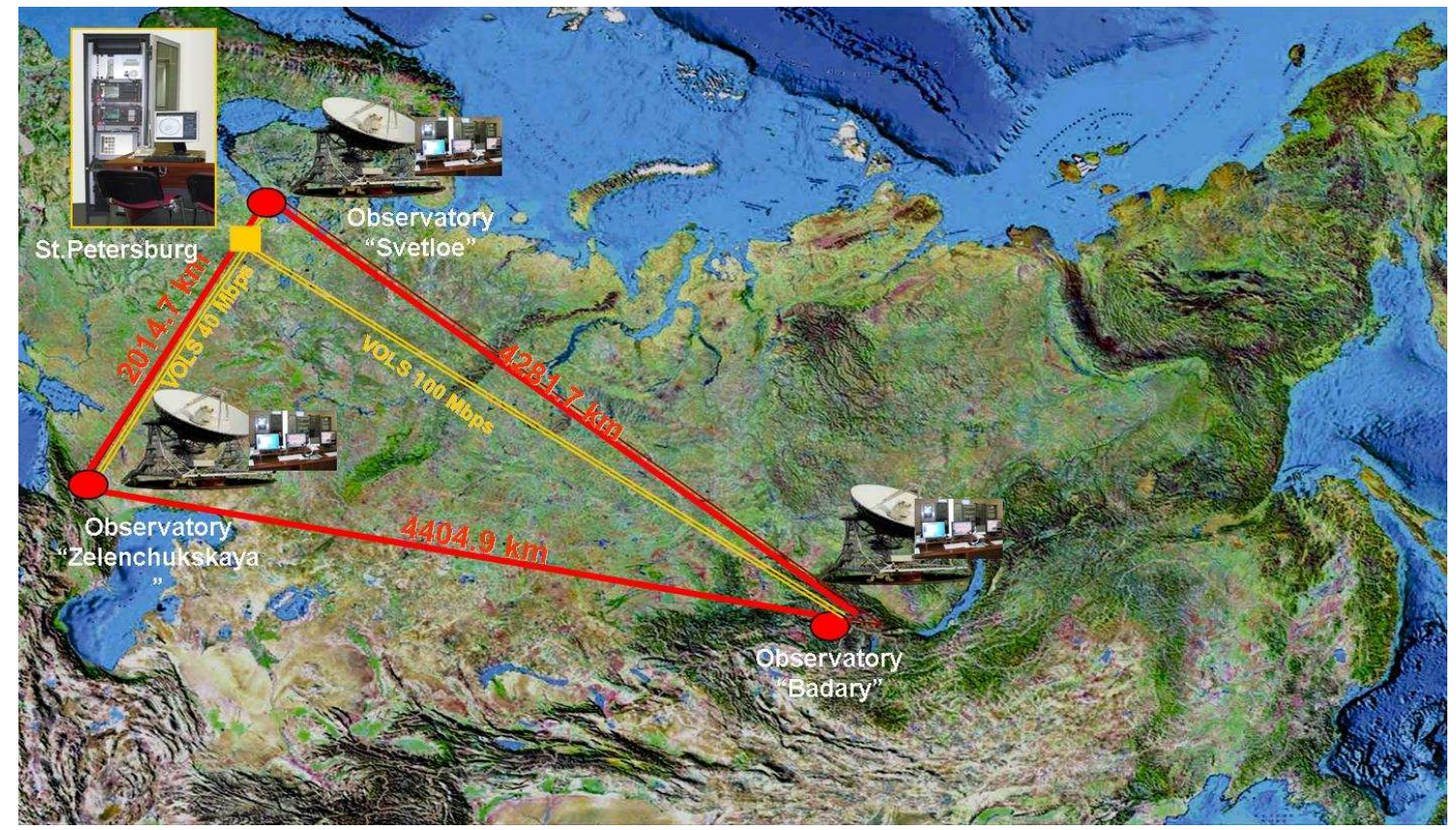

Figure 1: VLBI Network "Quasar"

This Network consists of three Russian radio astronomical observatories "Svetloe", "Zelenchukskaya" and "Badary" which make up three-element VLBI Network with baselines of about $2015 \times 4282 \times 4404 \mathrm{~km}$ assembling with the Control and Processing Center in St. Petersburg [1]. The guideline of the Russian VLBI Network "Quasar" is to carry out astrometrical and geodynamical investigations. The main part in the observational schedule is devoted to the international VLBI sessions with data processing at the Haystack or Bonn correlators. But since 2006, when the "Badary" station was put into operation, pure domestic observational programs are fulfilled with preliminary data processing at the IAA correlator. To maintain these geodynamical programs and to develop the whole system as well the e-VLBI technology is being developed and tested now at the IAA. This paper describes IAA activity in developing the real-time VLBI system using the high speed digital communication links.

\section{Communications}

In 2007 all observatories of the VLBI Network "Quasar" were linked by optical fiber lines (Fig.2) to provide both e-VLBI modes for determining Universal Time within Intensive 1-hour sessions and real-time remote monitoring each part of the Network. All observatories of the Network "Quasar" were equipped with UNIX servers for data buffering. Now the observatories 
have the so-called "last mile"communication channels at $100 \mathrm{Mbps}$ rate and via the Internet at 100, 40, 2 Mbps rate for the "Badary", "Zelenchukskaya" and "Svetloe" correspondingly.

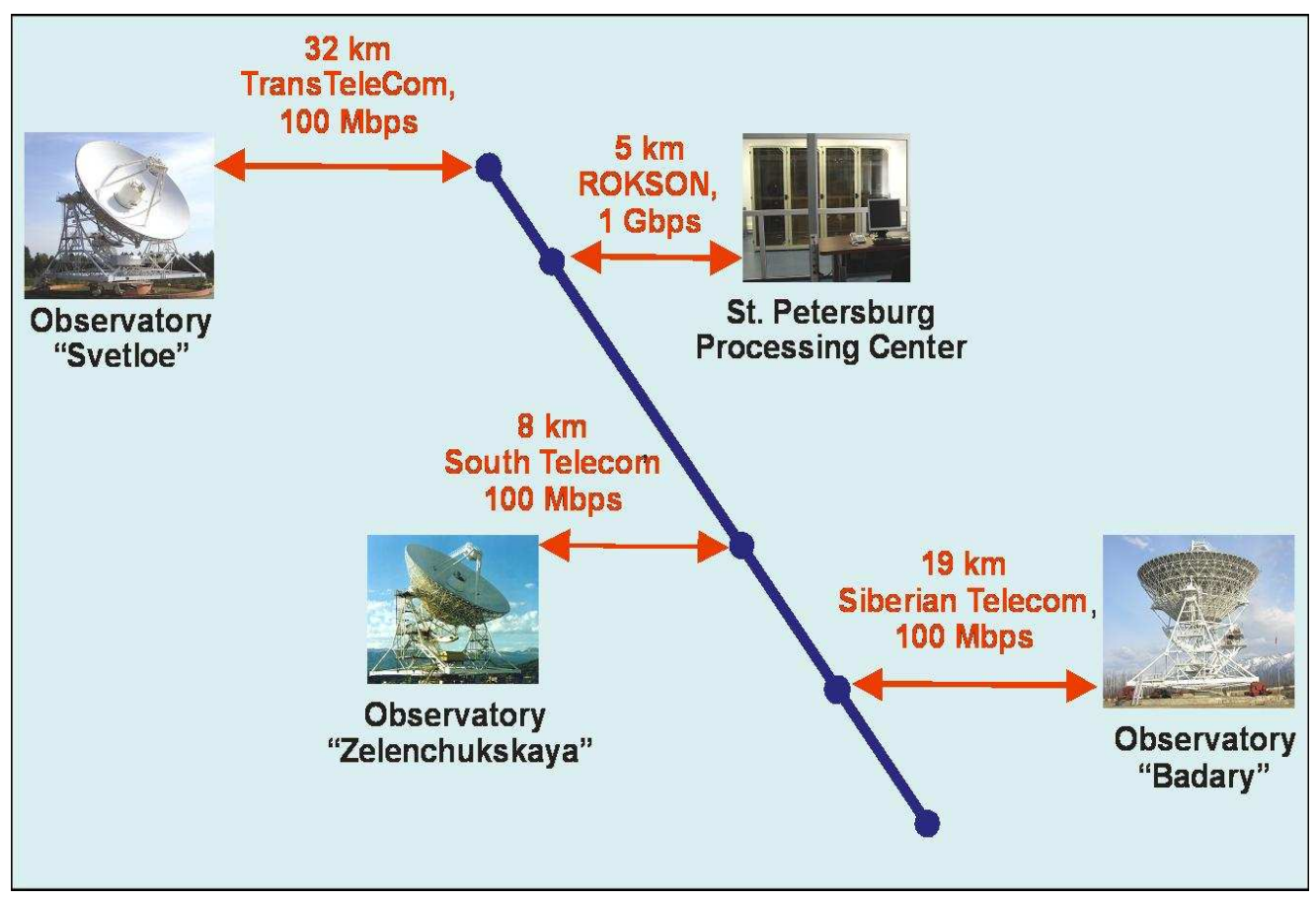

Figure 2: Optical Fiber Lines

\section{Experiments}

The first test of data transfer between the buffer server in IAA RAS and the Haystack Correlator was carried out using Tsunami-UDP protocol. The test scan of an Intensive session was transmitted via the Internet at 50Mbps (Fig.3).

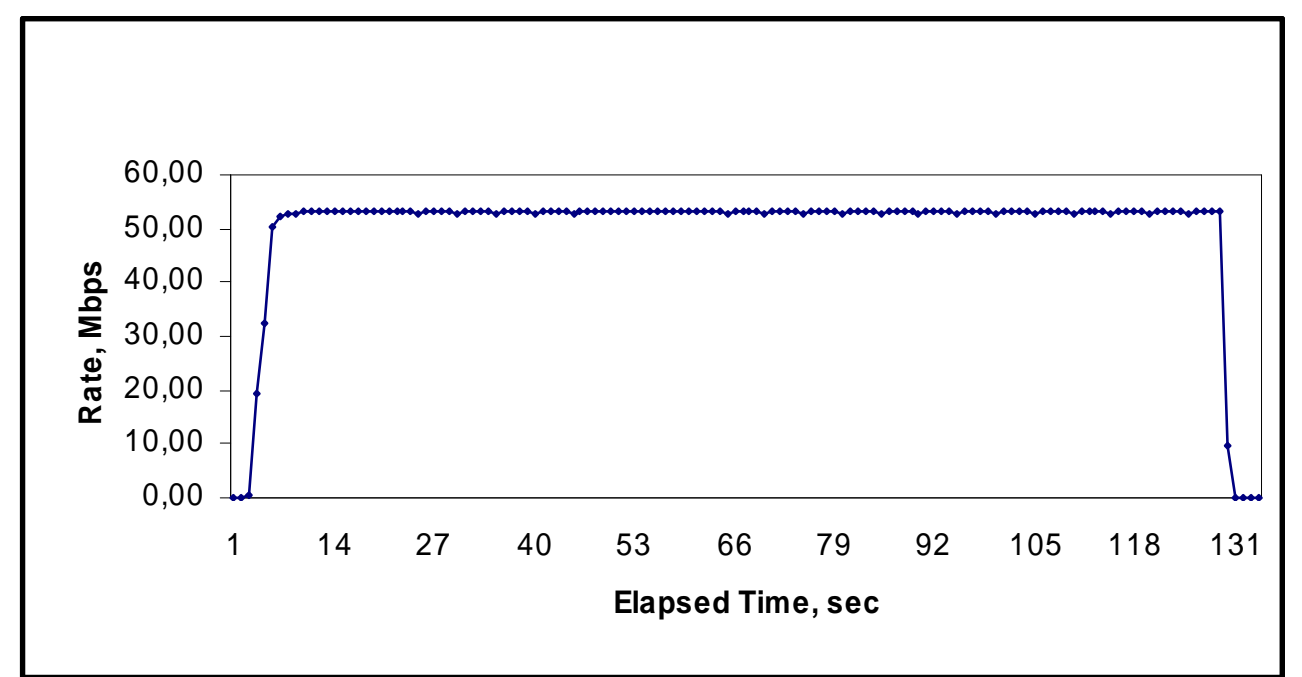

Figure 3: Transmission of a $0.5 \mathrm{~GB}$ scan from an Intensive session via the Internet (ISP RAS) using Tsunami- UDP protocol from St.Petersburg to Haystack. 
Intensive sessions for determining Universal Time using e-VLBI mode with the Network "Quasar" with baselines "Svetloe" - "Badary" and "Zelenchukskaya" - "Badary" were started in 2009 year. The near real-time e-VLBI mode has been used. VLBI observation data recorded with Mark5B were transmitted to the buffer server at the end of each 1-hour session (Fig.4).

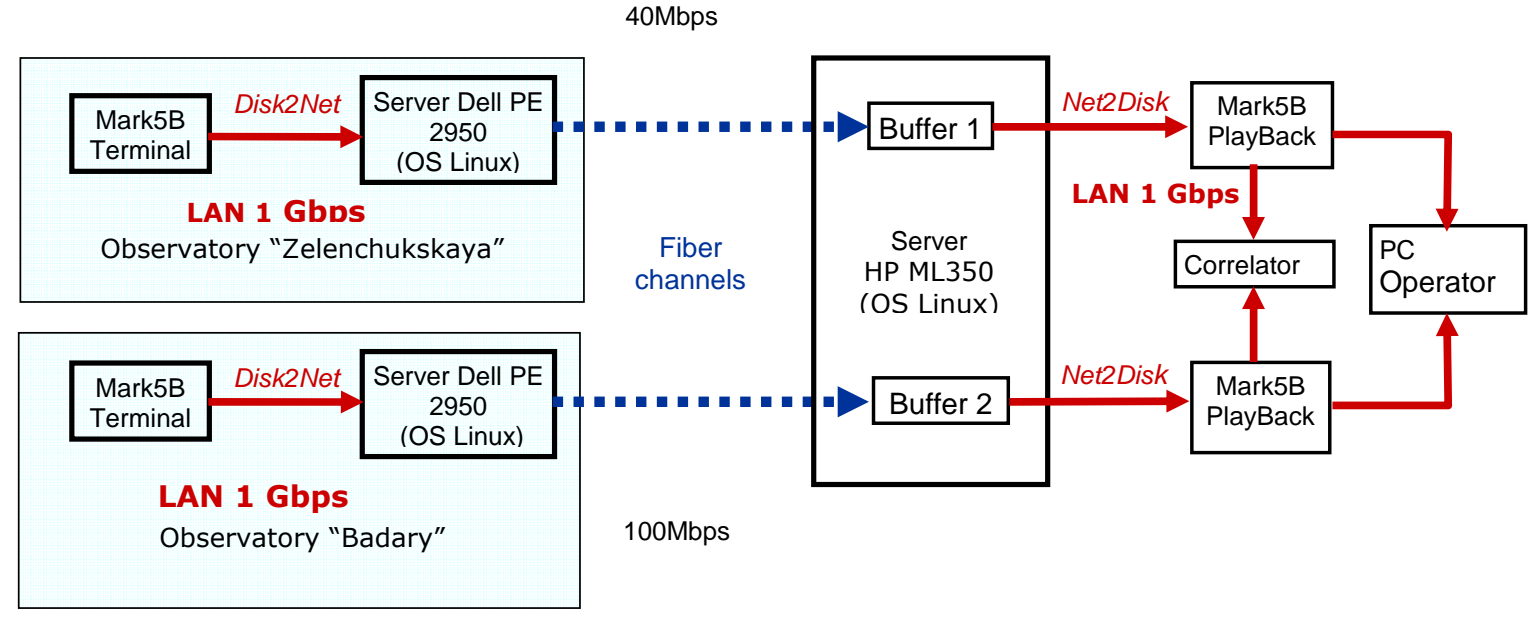

Figure 4: Algorithm of data transmission in e-VLBI mode. Mark5B - LAN - Buffer Server - Internet Buffer Server - LAN - Mark5B - Correlator. Network testing was realized with iperf (end-to-end).

Five transmissions of Intensive session data from "Zelenchukskaya" and "Badary" observatories to the IAA RAS Control and Processing Center at St.Petersburg have been done (Ru-Test038 - 18 April 2009, Ru-U056 - 24 April 2009, Ru-U057 - 7 May 2009, Ru-U058 13 May 2009 and Ru-U059 - 24 May 2009) [2]. Each of these Intensive sessions of $\sim 40$ GB were transmitted at 25-40 Mbps rate via the Internet using Tsunami-UDP protocol .

Figs.5 and 6 illustrate the transmission of a 2 GB scan of the Intensive session via the Internet from "Zelenchukskaya" and "Badary" observatories to the Control and Processing Center at St.Petersburg correspondingly.

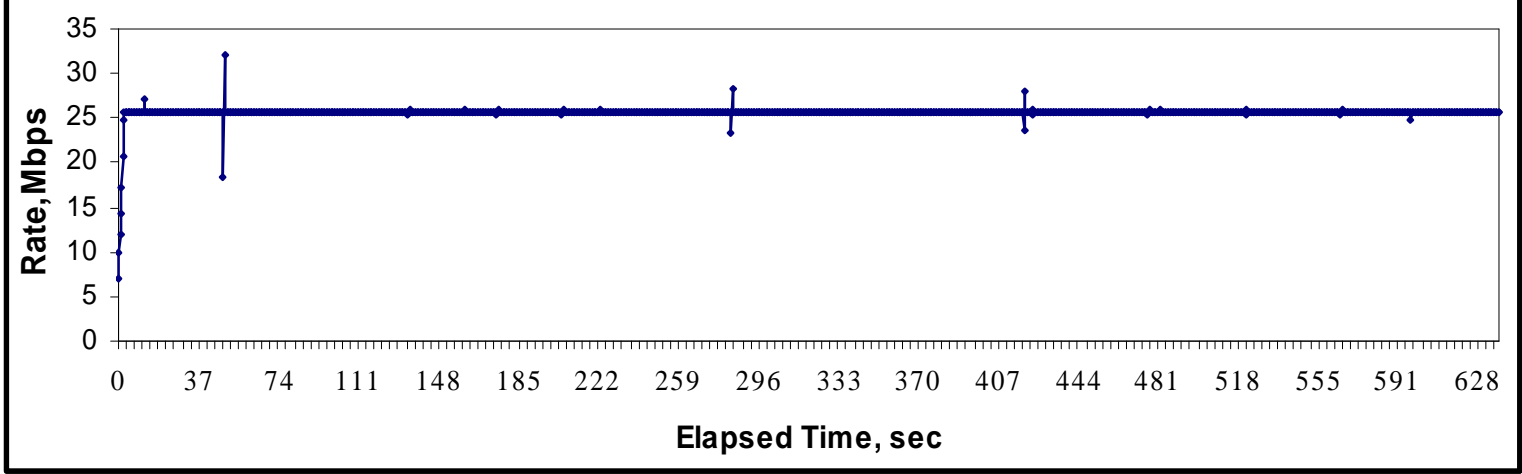

Figure 5: Transmission of a 2 GB scan of Intensive session via the Internet (ISP "South Telecom") using Tsunami-UDP Protocol from the "Zelenchukskaya" Observatory to St.Petersburg. 


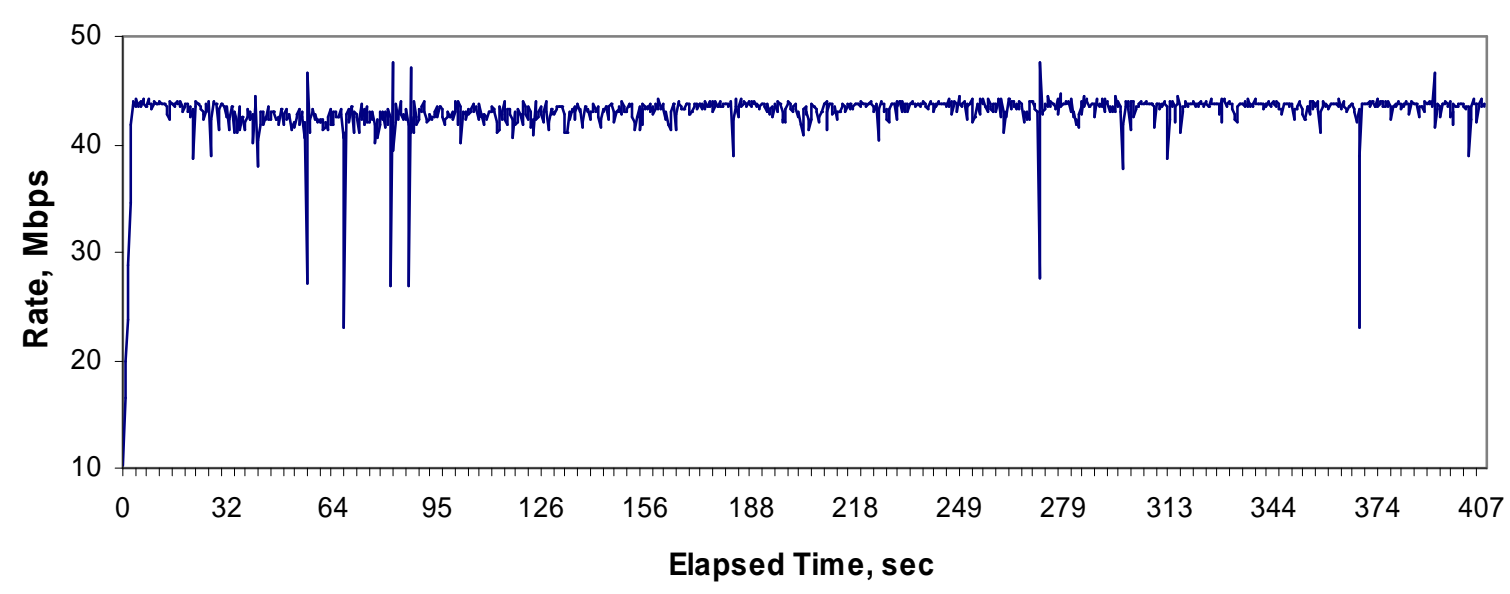

Figure 6: Transmission of a 2 GB scan of Intensive session via the Internet (ISP "Siberian Telecom") using Tsunami-UDP Protocol from the "Badary" Observatory to St.Petersburg.

The drop-outs and varying rates at figs.5 and 6 are due to the noise character of the public Internet chanals.

Fig.7 shows UT1-UTC differences of the IAA (Ru-U) and IERS 05 C04 series.

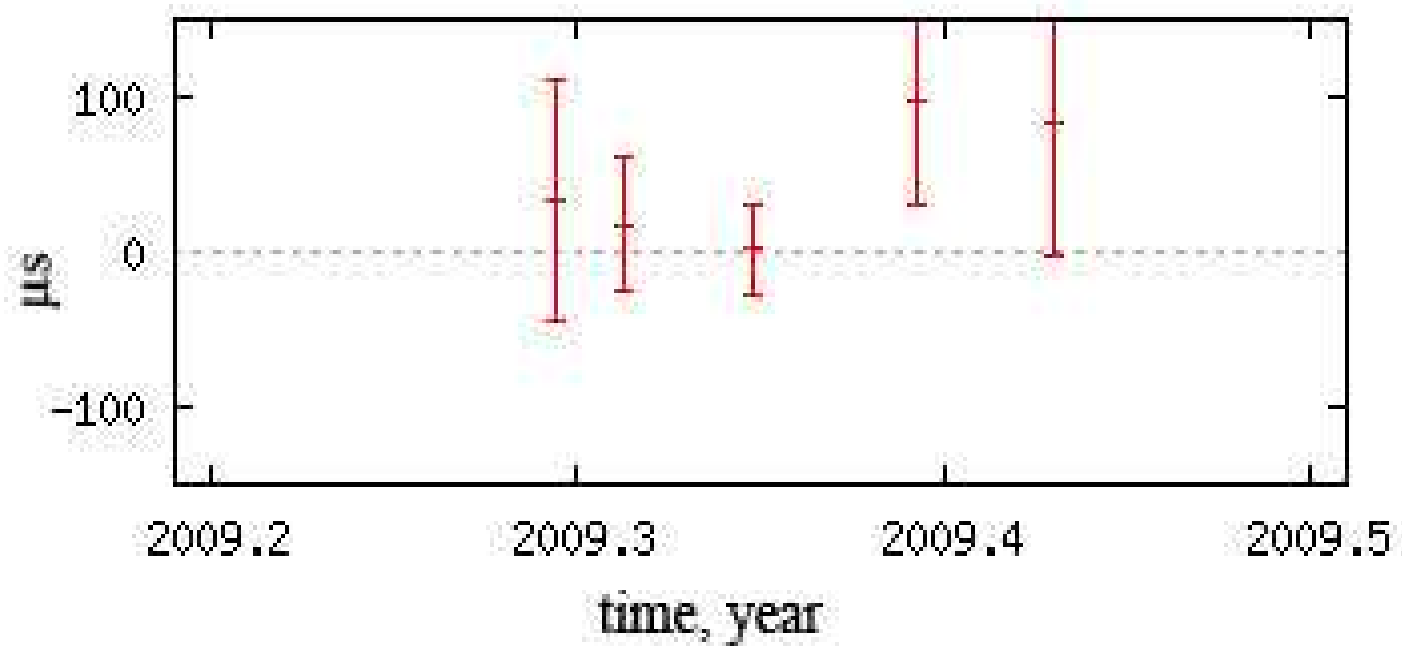

Figure 7: UT1-UTC Differences of IAA (Ru-U) and IERS 05 C04 for five e-VLBI sessions. RMS - 37 $\mu$ s

This allows us to draw a conclusion that the tested e-VLBI mode is reliable and could be used as regular e-VLBI mode on VLBI Network "Quasar". 


\section{Our nearest future}

We plan to develop an algorithm to automate the overall data transfer process from Mark5B recorders of observatories to the Control and Processing Center. By the end of 2009 the Network "Quasar" observatories and the Control and Processing Center of IAA will be supplied with hardware and software providing VLBI data transmission rates of at least $100 \mathrm{Mbps}$ from all observatories.

\section{References}

[1] A. Finkelstein, A. Ipatov, S. Smolentsev The Network "QUASAR": 2008-2011. "Measuring the Future". Proc. of the Fifth IVS General Meeting, A.Finkelstein, D. Behrend (eds.), "Nauka", St. Petersburg, 2008, 39-46.

[2] http://quasar.ipa.nw.ru/vlbi/?e=2009 